\title{
Evaluation of Omeprazole Limited Sampling Strategies to Estimate Constitutive Cytochrome P450 2C19 Activity in Healthy Adults
}

\author{
Swan Lin, PharmD' ${ }^{1}$, Mina Nikanjam, MD, Ph.D. ${ }^{1}$, Edmund V. Capparelli, PharmD1, \\ Alessandro Allegrini, MS ${ }^{2}$, Daniele Pavone, $\mathrm{MS}^{2}$, Dong-Seok Yim, MD, Ph.D. ${ }^{3}$, Muhammad \\ M. Hammami, MD, Ph.D. ${ }^{4}$, Joseph S. Bertino Jr., PharmD ${ }^{5}$, Anne N. Nafziger, MD, Ph.D. ${ }^{5}$, \\ Yoo-Sin Park, PhD ${ }^{6}$, Ophelia Q. Yin, Ph.D. ${ }^{7}$, and Joseph D. Ma, PharmD ${ }^{1}$ \\ ${ }^{1}$ University of California, San Diego, La Jolla, CA, USA \\ 2FOURPHARMA C.R.O. srl, Rome, Italy \\ ${ }^{3}$ Department of Pharmacology, Clinical Pharmacology Unit, and Clinical Trial Center/Clinical \\ Research Institute, Seoul National University College of Medicine and Hospital, Seoul, Korea \\ ${ }^{4}$ King Faisal Specialist Hospital \& Research Center, Riyadh, Saudi Arabia \\ ${ }^{5}$ Bertino Consulting, Schenectady, NY, USA \\ ${ }^{6}$ Shintong Rehabilitation Clinic, Suji-gu, Yongin-si, Gyeonggi-do, South Korea \\ ${ }^{7}$ School of Pharmacy, Faculty of Medicine, the Chinese University of Hong Kong, Shatin, New \\ Territories, Hong Kong.
}

\section{Abstract}

PURPOSE: Limited sampling strategy (LSS) is a validated method to estimate pharmacokinetic (PK) parameters from a reduced number of samples. Omeprazole is used to phenotype in vivo cytochrome P450 (CYP) 2C19 activity. This study examined a LSS using two estimation methods to determine apparent oral clearance (CL/F) and thus CYP2C19 activity.

METHODS: Data from 7 previously published studies included healthy subjects receiving a single, oral dose of omeprazole with intensive PK sampling. CL/F was estimated using noncompartmental analysis (NCA) and population PK modeling. LSS was simulated by selecting the 1, 2, 4, and/or 6-hour post dose time points. Linear regression was performed to assess whether $\mathrm{CL} / \mathrm{F}$ estimated from limited sampling could accurately predict CL/F from the full PK profile.

RESULTS: Median CL/F was $23.7 \mathrm{~L} / \mathrm{h}$ by NCA and $19.3 \mathrm{~L} / \mathrm{h}$ by population PK modeling. In comparing the LSS NCA estimated versus observed CL/F, all evaluated linear regression models had unacceptable coefficients of determination $\left(r^{2}\right.$, range: $\left.0.14-0.81\right)$. With the population PK approach, 737 plasma concentrations $(n=71)$ and CYP2C19 genotype data were described with a

Corresponding Author Joseph D. Ma, PharmD, FCP, UCSD, Skaggs School of Pharmacy and Pharmaceutical Sciences, 9500 Gilman Drive, MC 0714, La Jolla, CA, USA 92093-0714, TEL:1-858.822.3485, FAX: 1-858.822.6857, joema@ucsd.edu. Conflicts of Interest and Source of Funding

All authors declare no conflict of interest. Funding support was provided by a Research in Pediatric and Developmental Pharmacology NIH grant (1U54HD090259-01 to E.V.C.) 
one-compartment structural model with mixed zero and first order absorption and lag time. In comparing the population PK LSS estimated versus observed CL/F, all evaluated linear regression models had unacceptable $r^{2}$ (range: $0.02-0.74$ ). Post-hoc comparison of CYP2C19 poor metabolizers (PMs) versus CYP2C19 extensive metabolizers (EMs) resulted in significantly lower $\mathrm{CL} / \mathrm{F}$ in $\mathrm{PMs}$ versus EMs.

CONCLUSIONS: Omeprazole LSS performed poorly in estimating CL/F utilizing two separate estimation approaches and does not appear to be a suitable method for determining CYP2C19 activity.

\section{Keywords}

cytochrome P450; omeprazole; limited sampling strategy; CYP2C19

\section{Introduction}

Phenotyping is the preferred method to assess the clinical significance of a drug-drug interaction and to quantify real-time, in vivo cytochrome P450 (CYP) activity. Omeprazole is used as a probe drug to phenotype CYP2C19 and CYP3A activity ${ }^{1}$. Omeprazole apparent oral clearance (CL/F), metabolic ratios of omeprazole to 5-hydroxyomeprazole plasma concentrations, or area under the concentration time curve (AUC) ratios are phenotyping parameters used to evaluate CYP2C19 activity ${ }^{2}$. Intensive sampling of omeprazole and metabolite concentrations is performed to measure these phenotyping parameters and to calculate PK parameters by non-compartmental analysis (NCA). Intensive sampling can be cumbersome, as 10 to 16 blood samples are collected up to 24-hours post dose ${ }^{3}$.

Limited sampling strategy (LSS) is a validated method to estimate PK parameters from a reduced number of blood samples. Omeprazole LSS utilizing plasma concentrations and AUC ratios have been previously published ${ }^{4,5}$. However, study design limitations (i.e., small sample sizes) existed for previously reported studies ( $\mathrm{n}=18$ and 37) and complicate the ability to recommend such LSS models for general use. Beyond the NCA LSS approach, a LSS with a population PK approach has been used for a phenotyping cocktail study and a phenotyping study to quantify constitutive CYP3A activity ${ }^{6,7}$. With data from seven previously published studies in healthy adult subjects, the first objective was to develop novel omeprazole limited sampling models to estimate CL/F utilizing a NCA LSS approach. The second objective was to determine the feasibility of an omeprazole LSS utilizing a population PK approach for parameter estimations. Based on the known CYP2C19 genedose effect ${ }^{8}$, omeprazole $\mathrm{CL} / \mathrm{F}$ estimates were examined to determine if such estimates readily discriminate between CYP2C19 extensive metabolizers (EMs) and CYP2C19 poor metabolizers (PMs).

\section{Materials and Methods \\ STUDY SUBJECTS AND SAMPLING}

Institutional Review Board exemption was obtained for this study. Omeprazole plasma concentration data were obtained from seven studies in healthy adult subjects who received a single, oral 20 or $40 \mathrm{mg}$ dose with intensive PK sampling 0 to 24 hours post dose (Table 1, 
Supplemental Digital Content) ${ }^{3,8-13}$. Omeprazole and metabolite plasma concentration detection and genotyping methods for CYP2C19*1, *2, *3, and *17 alleles are described elsewhere ${ }^{3,8-13}$.

\section{NON-COMPARTMENTAL ANALYSIS FOR LIMITED SAMPLING STRATEGY}

Omeprazole CL/F was calculated using NCA with Phoenix ${ }^{\circledR}$ WinNonlin 7.0 (Cetara USA, Inc., Princeton, NJ, USA). Full concentration time profiles for all subjects were used to characterize omeprazole $\mathrm{AUC}_{\text {inf }}$ and CL/F (Figure 1, Supplemental Digital Content). Traditional LSS entailed randomizing subject data into training $(n=56)$ and validation $(\mathrm{n}=114)$ sets. The selected time points were $0,1,2,4$, and/or 6-hours post dose since these were observed across all studies (Table 1, Supplemental Digital Content) and were evaluated in a previous study ${ }^{5}$. Linear regression equations to estimate $\mathrm{CL} / \mathrm{F}$ as a function of partial AUCs 14,15 were derived from the training set. The estimated, log-transformed CL/F from LSS were compared with the observed CL/F calculated from the full concentration and time profiles using R version 3.2.2 (R Foundation, Vienna, Austria). Preset criterion for model selection was a coefficient of determination $\left(I^{2}\right) \geq 0.9^{16}$. The resulting equations were used to calculate individual $\mathrm{CL} / \mathrm{F}$ estimates from the validation set. If the model equations fulfilled $r^{2} \geq 0.9$, bias and precision were then determined by relative percent mean prediction error (\%MPE, $-5 \%$ to $5 \%$ ) and relative percent mean absolute error (\%MAE, $\leq 0 \%)^{16}$.

\section{LIMITED SAMPLING STRATEGY WITH A POPULATION PK APPROACH}

The full concentration time data of four studies $3,8,10,12$ (Table 1, Supplemental Digital Content) with available CYP2C19 genotype data $(\mathrm{n}=71)$ were modeled using NONMEM (version 7.3.0, GloboMax, Hanover, MD, USA) with a first-order conditional estimation method with interaction. An exponential error model was applied to describe inter-subject variability, and a mixed additive and proportional error model applied to describe the residual variability. Individual subject random effects and PK parameter estimates were generated by post hoc Bayesian estimation. Potential covariates were added univariately to the model on $\mathrm{CL} / \mathrm{F}$ and $\mathrm{V}$ as a linear function. Covariates that were significant (change in the objective function of $>3.84, \mathrm{p}<\sim 0.05)$ and improved the model fit were retained. Covariates were retained in the final model after multivariate screening with forward selection (change in objective function by $>7.88, \mathrm{p}<\sim 0.005$ ). To test whether estimated $\mathrm{CL} / \mathrm{F}$ from limited time points could be predictive of CYP2C19 activity, a final model that did not evaluate CYP2C19 activity as a potential covariate was selected, in order to simulate lack of prior knowledge of CYP2C19 activity. The bootstrap procedure ( $\mathrm{n}=1000)$ was performed using WINGS for NONMEM (UC San Francisco, San Francisco, CA, USA). Limited sampling datasets were derived using PK time points at 0, 1, 2, 4, and/or 6-hours. Estimated CL/F were then compared post hoc by linear regression to the CL/F estimated from the full profile. The same preset criterion of $r^{2} \geq 0.9$ and acceptable limits of \%MPE and \%MAE were used.

\section{EVALUATION OF OMEPRAZOLE GENE DOSE EFFECT}

A gene-dose effect in omeprazole PK has been previously reported ${ }^{8}$. To confirm that a gene-dose effect was observed in the current analyses, omeprazole CL/F was evaluated by

Ther Drug Monit. Author manuscript; available in PMC 2019 December 01. 
the Wilcoxon Rank Sum test between CYP2C19 PMs versus homozygous and heterozygous CYP2C19 EMs.

\section{Results}

\section{LIMITED SAMPLING STRATEGY WITH NON-COMPARTMENTAL ANALYSES}

Data from 170 subjects with 2272 omeprazole plasma concentrations was included (Figure 1, Supplemental Digital Content). Using the full concentration time profile, median observed $\mathrm{CL} / \mathrm{F}$ was $23.7 \mathrm{~L} / \mathrm{h}$ and $\mathrm{V}$ was 35.6L (Table 2, Supplemental Digital Content). Utilizing the training set $(\mathrm{n}=56)$, eight linear regression models were developed and all models had unacceptable $r^{2}$ (range: $0.14-0.81$, Table 1). A representative plot of the best model with an $r^{2}$ of 0.81 is illustrated in Figure 1a.

\section{LIMITED SAMPLING STRATEGY WITH A POPULATION PK APPROACH}

Omeprazole plasma concentrations $(n=737)$ from 71 subjects with CYP2C19 genotype data were described with a one-compartment structural model with mixed zero and first order absorption and lag time. The median observed CL/F was $19.3 \mathrm{~L} / \mathrm{h}$ and $\mathrm{V}$ was $23.7 \mathrm{~L}$ (Table 2, Supplemental Digital Content). Female sex and age were independent predictors of V. The final model described the data without significant bias (Figure 2, Supplemental Digital Content). Seven linear regression models were developed and all had unacceptable $r^{2}$ (range: $0.02-0.74$, Table 1). A representative plot of the best model, with $r^{2}$ of 0.74 , is illustrated in Figure 1b.

\section{OMEPRAZOLE CLEARANCE AND CYP2C19 ACTIVITY}

There were 62 CYP2C19 EMs and 9 CYP2C19 PMs. In 13 of 15 linear regression models, the LSS estimated CL/F in CYP2C19 PMs was significantly lower than CYP2C19 EMs (Table 1). The two regression models that failed to predict lower CL/F for CYP2C19 PMs used a single, 1-hour time point. Using a later single or a combination of later time point(s) allowed for better estimation of CL/F. Ad hoc evaluation of the CYP2C19 phenotype as a covariate of CL/F in population PK modeling estimated 48.7\% lower CL/F in PMs than EMs. CYP2C19 activity covariate on CL/F resulted in a significant objective function value drop of $26.0(\mathrm{p}<0.05)$ from the base model.

\section{Discussion}

This study was unable to develop an acceptable omeprazole LSS model to estimate CL/F with NCA. These results add to the conflicting literature that support $5,17,18$ and refute ${ }^{19}$ the suitability of an omeprazole LSS approach. Differences in sample size, selection of omeprazole phenotyping parameter, and method of model validation exist among studies that may explain these conflicting findings. The selection of CL/F as a function of a partial AUC was done since omeprazole AUC currently lacks adequate validation, and using the AUC ratio of omeprazole to 5-hydroxyomeprazole has limited generalizability. Niioka reported three limited sampling models of plasma concentrations to estimate omeprazole $\mathrm{AUC}^{5}$. In a separate analysis of external data sets but using the same limited sampling models ${ }^{5}$, each model had bias and lacked precision ${ }^{19}$. In another study, a three-time point 
limited sampling model accurately estimated the AUC ratio of omeprazole to 5-

hydroxyomeprazole ${ }^{4}$, but was only applicable to Caucasian CYP2C19*1/*1 subjects. To the best of our knowledge, this is the first LSS study to utilize an omeprazole CL/F as a function of a partial AUC to estimate CYP2C19 activity.

Limited sampling model selection and model validation approaches differed between the current study and previous studies. In the current study, model selection was determined by an $r^{2} \geq 0.9^{16}$. In other studies, model selection and model validation were primarily determined from the highest $r^{2}$ or correlation coefficient $(r)^{5,17}$. Selecting a limited sampling model based on the highest $r^{2}$ or $r$ may not be correct ${ }^{4}$ since neither the $r^{2}$ norr $r$ are measures of predictive performance ${ }^{20}$.

Although the structural population PK model was similar to another published model ${ }^{21}$, the current model was unable to accurately characterize the absorption phase. Several structural models were tested and a lag time was incorporated in the one-compartment first-order absorption and elimination model. Due to the variability in individual observed $\mathrm{T}_{\max }$, predicted peak concentrations did not match observed peak concentrations in the final model. None of the limited sampling models developed with the population PK approach met the preset $r^{2}$ criterion. This was unexpected given that the same approach was successful in a study that used midazolam limited sampling to accurately quantify constitutive CYP3A activity ${ }^{22}$. With the high variability in omeprazole absorption, the wide observed range of $\mathrm{T}_{\max }$ overlapped with the selected limited sampling time points. These early time points may not have accurately represented the elimination phase, and thus omeprazole CL/F estimates from the LSS population PK approach may be unreliable.

By NCA and population PK modeling, omeprazole CL/F differed significantly between CYP2C19 PMs and CYP2C19 EMs. The current study observed a 2.8-6.4 fold lower CL/F in CYP2C19 PMs versus EMs; a magnitude of the gene-dose effect consistent with previously published studies ${ }^{8,23}$. Only nine subjects were CYP2C19 PMs, with only the variant CYP2C19*2 and $* 3$ alleles tested. The contribution of additional CYP2C19 variant alleles on omeprazole PK is unknown, but is likely not clinically meaningful due to a low population frequency of $<1 \%{ }^{24}$.

\section{Conclusions}

Omeprazole is a probe drug used for determining CYP2C19 activity. NCA or population PK LSS for omeprazole do not appear to be predictive of CL/F estimated from full concentration-time profiles.

\section{Supplementary Material}

Refer to Web version on PubMed Central for supplementary material.

\section{Acknowledgements}

Presented in part at the 46th Annual Meeting of the American College of Clinical Pharmacology, San Diego, CA, USA, September 2017. Dr. Lin was a post-doctoral fellow with funding supported by Pfizer Global Research and Development and the UCSD, Skaggs School of Pharmacy and Pharmaceutical Sciences, in La Jolla, CA. 


\section{References}

1. Gonzalez HM, Romero EM, Chavez Tde J, et al. Phenotype of CYP2C19 and CYP3A4 by determination of omeprazole and its two main metabolites in plasma using liquid chromatography with liquid-liquid extraction. J Chromatogr B Analyt Technol Biomed Life Sci 2002;780(2):459465.

2. Streetman DS, Bertino JS, Jr., Nafziger AN. Phenotyping of drug-metabolizing enzymes in adults: a review of in-vivo cytochrome P450 phenotyping probes. Pharmacogenetics 2000;10(3):187-216. [PubMed: 10803676]

3. Cho JY, Yu KS, Jang IJ, et al. Omeprazole hydroxylation is inhibited by a single dose of moclobemide in homozygotic EM genotype for CYP2C19. Br J Clin Pharmacol 2002;53(4):393397. [PubMed: 11966672]

4. Lawson EB, Wu JC, Baldwin RM, et al. Omeprazole limited sampling strategies to predict area under the concentration-time curve ratios: implications for cytochrome P450 2C19 and 3A phenotyping. Eur J Clin Pharmacol 2012;68(4):407-413. [PubMed: 22009190]

5. Niioka T Clinical usefulness of limited sampling strategies for estimating AUC of proton pump inhibitors. Yakugaku Zasshi 2011;131(3):407-413. [PubMed: 21372537]

6. Nguyen TT, Benech H, Pruvost A, et al. A limited sampling strategy based on maximum a posteriori Bayesian estimation for a five-probe phenotyping cocktail. Eur J Clin Pharmacol 2016;72(1):39-51. [PubMed: 26490357]

7. Yang J, Patel M, Nikanjam M, et al. Midazolam single time point concentrations to estimate exposure and cytochrome P450 (CYP) 3A constitutive activity utilizing limited sampling strategy with a population pharmacokinetic approach. J Clin Pharmacol In press.

8. Yin OQ, Tomlinson B, Chow AH, et al. Omeprazole as a CYP2C19 marker in Chinese subjects: assessment of its gene-dose effect and intrasubject variability. J Clin Pharmacol 2004;44(6):582589. [PubMed: 15145965]

9. Allegrini A, Nuzzo L, Scaringi AT, et al. Bioequivalence study of two capsule formulations of omeprazole in healthy volunteers. Arzneimittelforschung 2008;58(8):385-388. [PubMed: 18807578]

10. Baldwin RM, Ohlsson S, Pedersen RS, et al. Increased omeprazole metabolism in carriers of the CYP2C19*17 allele; a pharmacokinetic study in healthy volunteers. Br J Clin Pharmacol 2008;65(5):767-774. [PubMed: 18294333]

11. Hussein RF, Lockyer M, Hammami MM. Bioequivalence assessment of two capsule formulations of omeprazole in healthy volunteers. Arzneimittelforschung 2007;57(2):101-105. [PubMed: 17396620]

12. Johnson MD, Hamilton CD, Drew RH, et al. A randomized comparative study to determine the effect of omeprazole on the peak serum concentration of itraconazole oral solution. J Antimicrob Chemother 2003;51(2):453-457. [PubMed: 12562722]

13. Rhim SY, Park JH, Park YS, et al. Pharmacokinetics and bioequivalence of $20 \mathrm{mg}$ omeprazole capsule in 24 healthy Korean male volunteers. Int J Clin Pharmacol Ther 2009;47(1):23-29. [PubMed: 19203529]

14. Masters JC, Harano DM, Greenberg HE, et al. Limited sampling strategy of partial area under the concentration-time curves to estimate midazolam systemic clearance for cytochrome P450 3A phenotyping. Ther Drug Monit 2015;37(1):84-89. [PubMed: 25004135]

15. Tai W, Gong SL, Tsunoda SM, et al. Evaluation of partial area under the concentration time curve to estimate midazolam apparent oral clearance for cytochrome P450 3A phenotyping. Drug Metabol Drug Interact 2013;28(4):217-223. [PubMed: 24114901]

16. Al-Khatib M, Shapiro RJ, Partovi N, et al. Limited sampling strategies for predicting area under the concentration-time curve of mycophenolic acid in islet transplant recipients. Ann Pharmacother 2010;44(1):19-27. [PubMed: 19996322]

17. Bottiger Y Use of omeprazole sulfone in a single plasma sample as a probe for CYP3A4. Eur J Clin Pharmacol 2006;62(8):621-625. [PubMed: 16791583] 
18. Niioka T, Uno T, Sugimoto K, et al. Estimation of CYP2C19 activity by the omeprazole hydroxylation index at a single point in time after intravenous and oral administration. Eur J Clin Pharmacol 2007;63(11):1031-1038. [PubMed: 17701405]

19. Lam LH, Allegrini A, Hammami MM, et al. Evaluation of omeprazole limited sampling strategy to estimate cytochrome P450 (CYP) 2C19 activity in healthy adults. Clinical Pharmacology and Drug Development 2015;4(S1):26.

20. Sheiner LB, Beal SL. Some suggestions for measuring predictive performance. J Pharmacokinet Biopharm 1981;9:503-512. [PubMed: 7310648]

21. Marier JF, Dubuc MC, Drouin E, Alvarez F, Ducharme MP, Brazier JL. Pharmacokinetics of omeprazole in healthy adults and in children with gastroesophageal reflux disease. Ther Drug Monit 2004;26(1):3-8. [PubMed: 14749542]

22. Yang J, Patel M, Nikanjam M, et al. Midazolam single timepoint concentrations to estimate exposure and cytochrome P450 (CYP) 3A constitutive activity utilizing limited sampling strategy with a population pharmacokinetic approach. Journal of Clinical Pharmacology In press 2018.

23. Sakai T, Aoyama N, Kita T, et al. CYP2C19 genotype and pharmacokinetics of three proton pump inhibitors in healthy subjects. Pharm Res 2001;18(6):721-727. [PubMed: 11474773]

24. Zhou Y, Ingelman-Sundberg M, Lauschke VM. Worldwide distribution of cytochrome P450 alleles: a meta-analysis of population-scale sequencing projects. Clin Pharmacol Ther 2017;102(4):688-700. [PubMed: 28378927] 

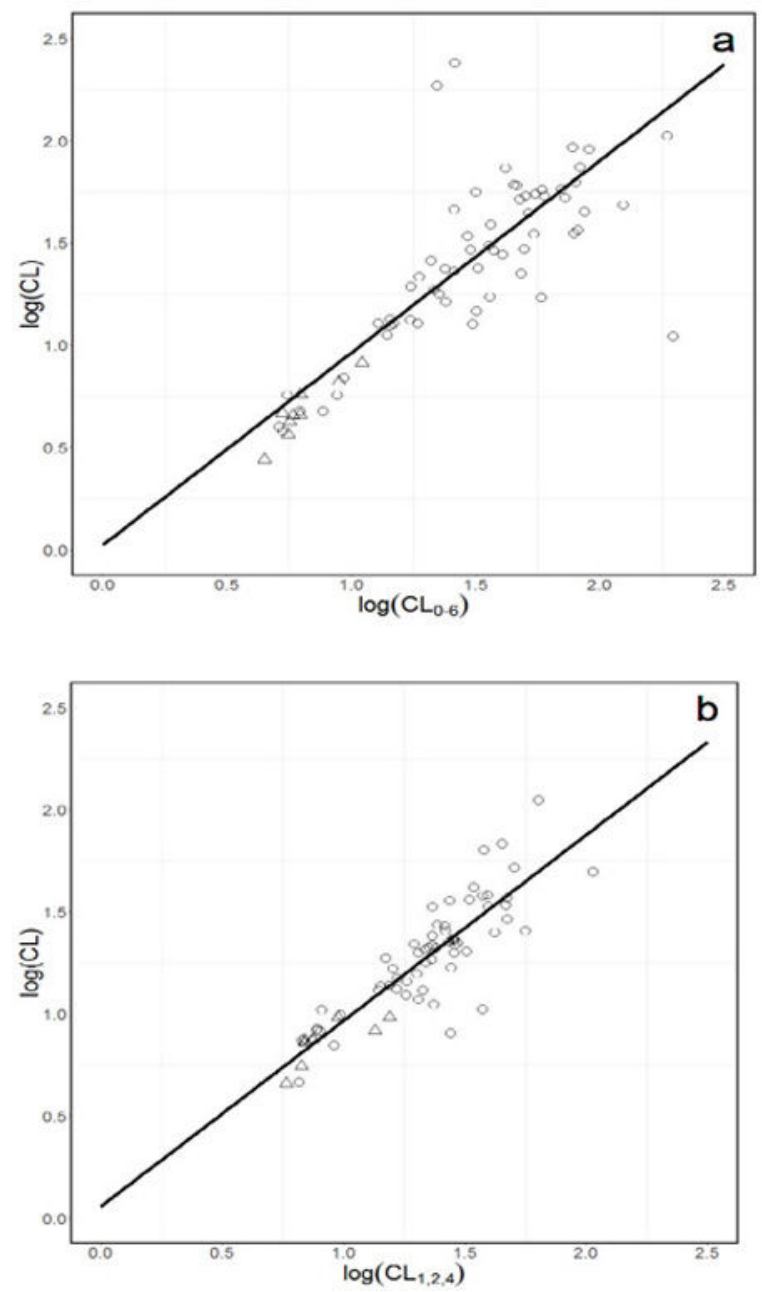

Figure 1.

Representative full concentration-time $\log (\mathrm{CL})$ versus limited sampling $\log (\mathrm{CL})$ plots for a. NCA model of $\log (\mathrm{CL})=0.023+0.94 \times \log \left(\mathrm{CL}_{0-6}\right)$ and b. Population PK model of $\log (\mathrm{CL})$ $=0.057+0.91 \times \log \left(\mathrm{CL}_{1,2,4}\right)$. Open circles represent 1 or 2 active $\mathrm{CYP} 2 \mathrm{C} 19$ alleles and open triangles represent 0 active CYP2C19 alleles. 
